Acknowledgments. We thank the Luohu Center for Disease Control (CDC), Futian CDC, Baoan CDC, Nanshan CDC, Longgang CDC, Yantian CDC, Longhua CDC, Guangming CDC, Pingshan CDC, Dapeng CDC, Shenzhen Samii Medical Center and Shenzhen Hezheng Hospital, all in Shenzhen, China, for offering 14-day isolation services for the discharged patients. We thank the Third People's Hospital of Shenzhen for the admission and treatment for the relapse patients.

Financial support. D.H. was supported by General Research Fund (grant no. 15205119) of Research Grants Council of Hong Kong and an Alibaba (China)-Hong Kong Polytechnic University Collaborative Research project. The funding agencies had no role in the design and conduct of the study; collection, management, analysis, and interpretation of the data; preparation, review, or approval of the manuscript; or decision to submit the manuscript for publication.

\section{References}

1. China National Health Commission. Diagnosis and treatment of the novel coronavirus (2019-nCoV) pneumonia in China [in Chinese]. China National Health Commission website. http://www.nhc.gov.cn/yzygj/s7653p/202002/ d4b895337e19445f8d728fcafle3e13a.shtml. Published 2020. Accessed April 16, 2020.

2. Lan L, Xu D, Ye G, et al. Positive RT-PCR test results in patients recovered from COVID-19. JAMA 2020. doi: 10.1001/jama.2020.2783.

3. Report of the WHO-China Joint Mission on Coronavirus Disease 2019 (COVID-19) from 16-24 February 2020. World Health Organization website. https://www.who.int/docs/default-source/coronaviruse/who-china-joint-missionon-covid-19-final-report.pdf. Published 2020. Accessed April 15, 2020.

4. Guan WJ, Ni ZY, Hu Y, et al. Clinical characteristics of coronavirus disease 2019 in China. N Engl J Med 2020. doi: 10.1056/NEJMoa2002032.

Conflicts of interest. D.H. was supported by an Alibaba (China)-Hong Kong Polytechnic University Collaborative Research project. All other authors declared no competing interests related to this article.

\title{
Pasteurized blood samples for transfusion compatibility testing during the coronavirus disease 2019 outbreak
}

\author{
Run Yao MS ${ }^{1}$, Yamei Shen BS ${ }^{1}$, Ying Tan MD, Pengcheng Zhou MD², Bijuan Li PhD¹, Xuegong Fan PhD²,3 and Ning Li PhD \\ ${ }^{1}$ Department of Blood Transfusion, Xiangya Hospital, Central South University, Changsha, 410007, Hunan, China, ${ }^{2}$ Department of Infectious Diseases, Xiangya \\ Hospital, Central South University, Changsha, China and ${ }^{3}$ Key Laboratory of Viral Hepatitis, Hunan Province, Changsha, China
}

To the Editor-In December 2019, a novel coronavirus pneumonia (COVID-19) was reported in Wuhan, China. As of April 2, 2020, 82,774 confirmed cases had been reported in China and 874,995 confirmed cases had been reported in other countries. No vaccine or antiviral therapeutics are yet available to prevent or treat COVID-19. ${ }^{1}$ Preventing infection is the current priority for disease control.

The SARS-CoV-2 virus is transmitted from person to person through droplets or direct contact. ${ }^{2}$ However, non-respiratory samples are also potential sources of COVID-19 infection. ${ }^{3}$ Virus-laden aerosols generated from blood-sample centrifugation pose risks for laboratory staff and broader nosocomial transmission. ${ }^{3,4}$ Traditional precautionary measures for infectious-sample processing include tertiary protection and operating in the biological safety cabinet. Preventive resources have been limited during this multiregional outbreak, posing huge risks to laboratory staff. Therefore, effective methods to ensure the safety of laboratory staff in low-resource settings are needed.

Pasteurization at $56^{\circ} \mathrm{C}$ for 30 minutes has been recommended to inactivate coronavirus, which might decrease the infectivity of samples and aerosols. To reduce infections and ensure safe and effective transfusion, we investigated the effects of pasteurization on transfusion compatibility testing.

Author for correspondence: Li Ning, E-mail: liningxy@csu.edu.cn

Cite this article: Yao R, et al. (2020). Pasteurized blood samples for transfusion compatibility testing during the coronavirus disease 2019 outbreak. Infection Control \& Hospital Epidemiology, 41: 1112-1114, https://doi.org/10.1017/ice.2020.138

\section{Methods}

Blood samples were collected from Xiangya Hospital, Central South University. Each sample was divided into 2 groups, an experimental group and a control group. Experimental samples were treated by pasteurization. The results of blood-group typing, irregular antibody screening, and cross-matching were compared between these 2 groups. Finally, samples of suspected SARS-CoV-2 were treated with pasteurization. Treated samples were used to test transfusion compatibility. Patients with suspected COVID-19 then received red blood cell (RBC) transfusion, and the effectiveness and safety of these transfusion were evaluated.

\section{Results}

The agglutination intensities of $\mathrm{A}, \mathrm{B}$ antigens and anti-A, anti-B antibodies of the samples in the 2 groups were $4+$. The forward and reverse types were consistent in the ABO blood group. In the $\mathrm{Rh}$ blood group, the agglutination intensity of $\mathrm{D}$ antigen was reduced from $4+$ to between $2+$ and $3+$ after heat treatment (Fig. 1). Regarding the effect of heat treatment on irregular antibody screening, our results showed that the response pattern of panel cells remained unchanged after heat treatment when the agglutination intensity was negative(-), uncertain( \pm ) or zero, and $1+, 2+$, or $3+$, respectively. However, the agglutination intensities of samples rating $4+$ were reduced to $3+$ after heat treatment (Fig. 2). Finally, no effect of heat treatment on the primary crossmatching was observed.

Our results indicated that heat treatment did not affect the results of transfusion compatibility testing. The RBC transfusion 


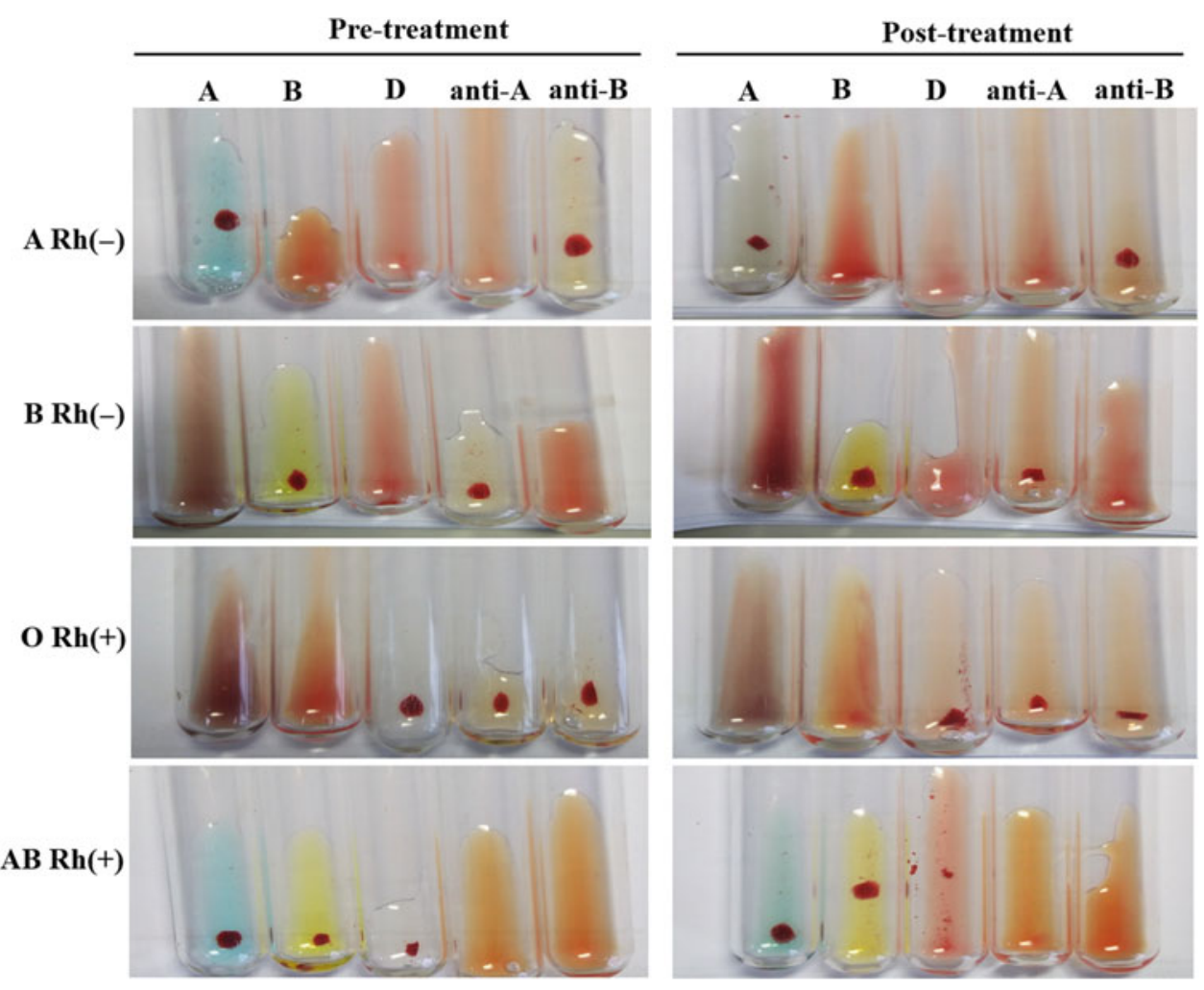

Fig. 1. The results of blood group typing.

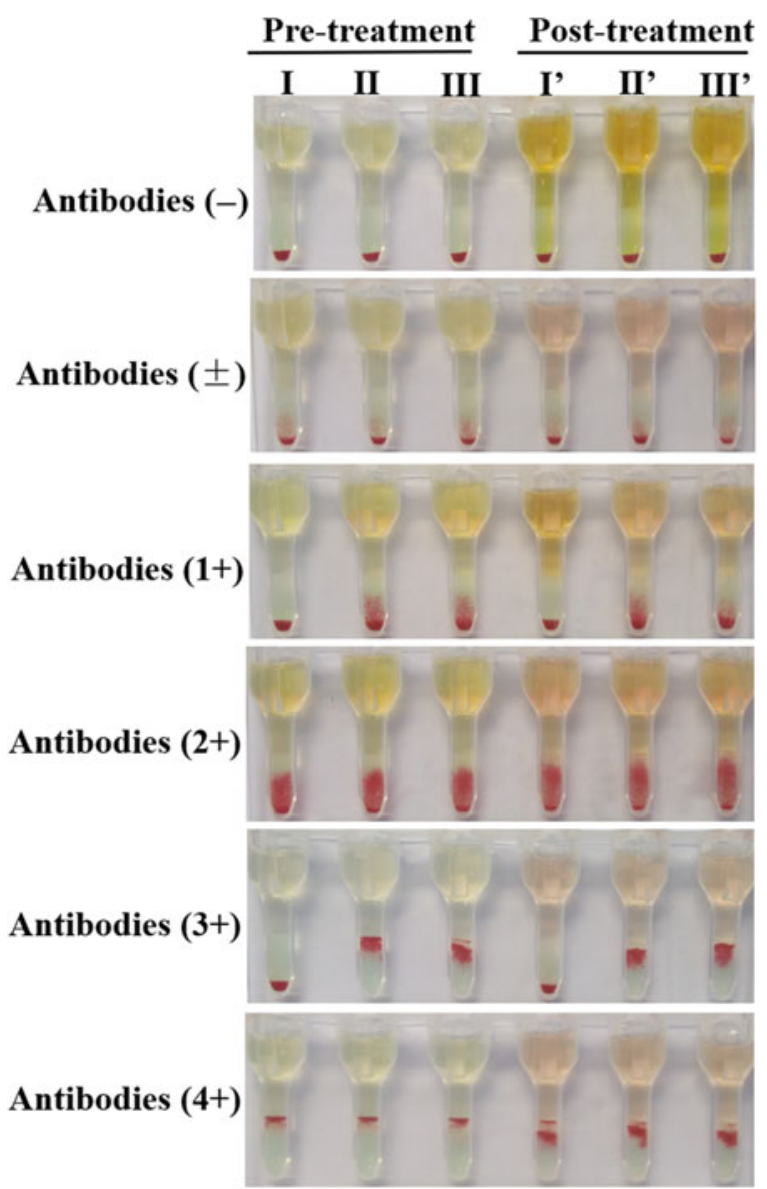

Fig. 2. The results of irregular antibodies screening. I, II, III represent panel cells no. 1, no. 2, and no. 3, respectively (Shanghai Blood Biomedical, Shanghai, China). 
in patients were safe and effective based on elevated 24-hour hemoglobin results or improved symptoms, with no hemolytic reactions or other adverse transfusion reactions. ${ }^{5}$

\section{Discussion}

We have demonstrated that pasteurization did not affect the results of transfusion compatibility testing and that blood transfusion based on this improved testing were safe and effective. Because the heat-inactivation method was simple, efficacious, and costeffective, it could be employed for the protection of laboratory staff, especially in resource-poor regions during the COVID-19 pandemic.

Since virus activity testing was not available in our laboratory, we were unable to determine whether the virus can still be contagious after thermal inactivation. Reports indicated that SARS-CoV-2 was sensitive to heat and thermal inactivation could efficiently eliminate the coronavirus infectivity. ${ }^{6}$ Heat treatment causes RBCs to rupture and form RBC fragments, which may have affected the detection results. Especially in gel microcolumns, false-positive results are likely. Therefore, the classic test-tube method should be used to instead of blood-type cards to perform blood-group typing of the heat-treated samples. However, irregular antibody screening and cross-matching could be performed using the anti-human-globulin card method.

In conclusion, during the COVID-19 pandemic, pasteurization can be used to test transfusion compatibility, to protect laboratory staff from infected samples, and to ensure safe and effective transfusion. Moreover, pasteurization is convenient and quick and suitable for use in hospitals.

\section{Acknowledgments. None.}

Financial support. This study was supported by the National Natural Science Foundation of China (grant no. 81873574) and by the Emergency Special Science and Technology Plan Project of COVID-19 Epidemic of Changsha City (grant no. 37935).

Conflicts of interest. All authors report no conflicts of interest relevant to this article.

\section{References}

1. She J, Jiang J, Ye L, et al. 2019 novel coronavirus of pneumonia in Wuhan, China: emerging attack and management strategies. Clin Transl Med 2020;9:19.

2. Chan JF, Yuan S, Kok KH, et al. A familial cluster of pneumonia associated with the 2019 novel coronavirus indicating person-to-person transmission: a study of a family cluster. Lancet 2020. doi: 10.1016/S0140-6736(20)30154-9.

3. Judson SD, Munster VJ. Nosocomial transmission of emerging viruses via aerosol-generating medical procedures. Viruses 2019;11(10): pii: E940.

4. Ong SWX, Tan YK, Chia PY, et al. Air, Surface environmental, and personal protective equipment contamination by severe acute respiratory syndrome coronavirus 2 (SARS-CoV-2) from a symptomatic patient. JAMA 2020. doi: $10.1001 /$ jama.2020.3227.

5. Carson JL, Grossman BJ, Kleinman S, et al. Red blood cell transfusion: a clinical practice guideline from the AABB. Ann Intern Med 2012;157:49-58.

6. Rabenau HF, Cinatl J, Morgenstern B, et al. Stability and inactivation of SARS coronavirus. Med Microbiol Immunol 2005;194(1-2):1-6.

\title{
India fights hard to neutralize the spread of COVID-19
}

\author{
Govindasamy Agoramoorthy PhD \\ College of Pharmacy and Health Care, Tajen University, Yanpu, Pingtung, Taiwan
}

To the Editor-A novel coronavirus (SARS-CoV-2) that originated in Wuhan, China, has created a pandemic across 198 countries over the first few months of 2020. ${ }^{1}$ As of April 5, 2020, India has 3,072 confirmed cases, 213 recovered persons, and 75 deaths, and more new cases are emerging rapidly. India has a huge population of $>1.3$ billion people, and cities such as Delhi, Mumbai, Kolkata, Chennai, Bangalore, Hyderabad, and Pune harbor millions of people who rely on public transportation. The government has aggressively promoted social distancing to minimize the spread of this virus.

On a daily basis, millions of people pass through crowded train stations such as Delhi, Howrah, Sealdeha, Mumbai, and Chennai. For example, the Sealdha station alone receives 1.8 million passengers, and most are from low- and middle-income families that depend on intracity transportation. Such close contact among people in highly crowded areas is potentially catastrophic for

\footnotetext{
Author for correspondence: Govindasamy Agoramoorthy, E-mail: agoram@tajen. edu.tw

Cite this article: Agoramoorthy G. (2020). India fights hard to neutralize the spread of COVID-19. Infection Control \& Hospital Epidemiology, 41: 1114-1115, https://doi.org/ $10.1017 /$ ice. 2020.140
}

community spread of the virus. In response to this crisis, the government has created expert groups to tackle the practical problems on the ground. For example, both international and domestic flights have been grounded. ${ }^{2}$

Few detection centers to screen for SARS-CoV-2 currently exist, so a transportation chain is necessary to take samples (eg, sputum, blood, urine, and nasal swabs) from collection points to testing centers. Several days are required to obtain test results. In addition, false-positive and false-negative results can occur and must be carefully avoided. The country's elite Indian Council of Medical Research should create more detection and observation centers to facilitate a more rapid testing process. Through agencies such the National Institute of Virology in Pune, the government has tried to bring factual awareness regarding the virus and to eliminate the spread of false information via social media. However, this effort needs support from all healthcare NGOs to encourage people to remain calm and to act rationally.

India's pharmaceutical industries are also facing difficulties because they obtain $70 \%$ of all active pharmaceutical ingredients from neighboring China, where the pandemic originally started. ${ }^{3}$ In addition, pharmaceutical trading companies depend on 